gewöhnlicher blauer Tinte schreibe, ist sein Inhalt wahr. Ist er mit roter Tinte geschrieben, ist er falsch.« Nach einem Monat erhalten seine Freunde den ersten mit blauer Tinte geschriebenen Brief: »Hier ist alles ganz wunderbar. Die Geschäfte sind voller Waren, Lebensmittel gibt es reichlich, die Wohnungen sind groß und ordentlich geheizt, die Kinos zeigen Filme aus dem Westen, und es gibt viele hübsche Mädchen, die auf eine Affäre aus sind - das einzige, was man nicht bekommen kann, ist rote Tinte ...«

Zizek knüpft an diesen Witz die Frage an: »Ist das nicht genau das Grundmuster, nach dem Ideologie funktioniert? Nicht nur unter ,totalitärer' Zensur, sondern vielleicht auch unter den verfeinerten Verhältnissen liberaler Zensur? Wir ,fühlen uns frei', weil uns die Sprache fehlt, unsere Unfreiheit auszudrücken. Die fehlende rote Tinte bedeutet heute, dass alle wesentlichen Begriffe, die wir gebrauchen, um den gegenwärtigen Konflikt zu charakterisieren - ,Krieg gegen den Terror', ,Menschenrechte' und so weiter -, falsche Begriffe sind, die unsere Wahrnehmung der Situation mystifizieren, anstatt den Gedanken zuzulassen: Unsere ,Freiheiten' selbst verdecken unsere tiefere Unfreiheit und erhalten sie. Das glei- che gilt für die uns angetragene Wahl zwischen ,Demokratie oder Fundamentalismus'.«

Angesichts der Anmerkungen dieses slowenischen Zeitgenossen drängt sich die Frage auf, in welcher Farbe eigentlich die Redetexte unserer Politiker geschrieben sind.

Wo aber bleibt, um mit Erich Kästner zu sprechen, wo aber bleibt am Ende nun das Positive? Eine schwierige Frage, die, fürchte ich, sich einer kurzen und schneidigen Antwort entzieht. Ein Fingerzeig indes lässt sich erkennen: Die deutschen Bischöfe nämlich haben einen ganz einfachen, präzisen, unmissverständlichen Satz geprägt, und dieser Satz lautet: Gerechtigkeit schafft Frieden. Sie führen dazu aus: »Das Leitbild des gerechten Friedens beruht auf einer letzten Endes ganz einfachen Einsicht: Eine Welt, in der den meisten Menschen vorenthalten wird, was ein menschenwürdiges Leben ausmacht, ist nicht zukunftsfähig. Sie steckt auch dann voller Gewalt, wenn es keinen Krieg gibt. Verhältnisse fortdauernder schwerer Ungerechtigkeit sind in sich gewaltgeladen und gewaltträchtig. Daraus folgt positiv: ,Gerechtigkeit schafft Frieden'.«

Jürgen Hafemann

\title{
Präventives Konfliktmanagement im Zusammenhang mit der Umsetzung der Europäischen Wasserrahmenrichtlinie im Flusseinzugsgebiet der Oder
}

\author{
Jürgen Hafemann, Dr., Institut \\ für Friedensforschung und Si- \\ cherheitspolitik an der Univer- \\ sität Hamburg (IFSH)
}

Die »Petersberger Erklärung « $(\mathrm{PE})^{1}$ vom März 1998 bezeichnet gemeinsame Visionen als Schlüsselfaktor für ein konfliktarmes und effektives Management internationaler Wasserressourcen. Der bevorstehende Beitritt der Republiken Polen und Tschechien zur Europäischen Gemeinschaft stellt die anspruchsvolle Aufgabe, solche gemeinsamen Visionen mit Ländern zu teilen, die unterschiedlich entwickelte Volkswirtschaften, ungleich gewachsene politische Systeme und Verwaltungsstrukturen, verschiedene Rechtstraditionen, Standards des Umweltschutzes sowie divergierende Formen ökologischen Bewusstseins in die Gemeinschaft einbringen. Je deutlicher die Unterschiede der Anrainerstaaten hinsichtlich ihrer politischen Systeme und gesellschaftspolitischen Zielvorstellungen, des wirtschaftlichen Entwicklungsniveaus und ihrer infrastrukturellen Kapazitäten sind, desto größer sind die Anforderungen an ein konsensfähiges grenzüberschreitendes Gewässermanagement.

Mit ihrer Wasserrahmenrichtlinie (WRRL) ${ }^{2}$ haben die bishe-

1 Vgl. 1. Petersberger Gespräch »Globale Wasserpolitik« Petersberg bei Bonn, 3. - 5. März 1998: Petersberger Erklärung, Entwicklungspolitisches Forum/DSE, Berlin 1998

2 Siehe Amtsblatt der Europäischen Gemeinschaften v. 22.12.2000, L 327, rigen Mitgliedsstaaten der EU eine gemeinsame Vision von nachhaltiger Nutzung europäischer Wasserressourcen in ein strategisches Aktionsprogramm für die kooperative Bewirtschaftung auch der grenzüberschreitenden Gewässer umgesetzt. Die Vielzahl bisher geltender Wasserrichtlinien wurde durch ein einheitliches und modernes europäisches Wasserrecht ersetzt. Gelten wird dieses Recht auch für die künftigen Mitgliedsländer der EU - freilich ohne dass sie am Entwurf dieser Vision mitwirken konnten.

Die wichtigsten Elemente der WRRL ${ }^{3}$ :

1-22. Auch: http://www.tu-harburg.de/ wwven/eu-wrrl.htm. Die »Richtlinie 2000/60 des Europäischen Parlaments und des Rates vom 23.10.2000 zur Schaffung eines Ordnungsrahmens für Maßnahmen der Gemeinschaft im Bereich der Wasserpolitik « (WRRL) trat am 22.12.2000 in Kraft und bündelt die bisher auf über 30 EU-Richtlinien verteilten Vorgaben zu Gewässerbewirtschaftung in der EU. Die zwei wichtigsten Ziele der WRRL:

1. Schaffung eines Ordnungsrahmens für die Europäische Wasserwirtschaft und Bündelung des wasserwirtschaftlichen Handelns in Maßnahmeprogrammen bzw. Bewirtschaftungsplänen bis 2009 .

2. Erreichung eines guten Gewässerzustandes (aller Oberflächengewässer und des Grundwassers) in allen Ländern der EU innerhalb von 15 Jahren.

3 Vgl. hierzu: Moss, Timothy: Die EU-Wasserrahmenrichtlinie als Beispiel eines Institutionenwandels: Forschungsbedarf und Erklärungsansätze aus politik- und raumwissenschaftlicher Sicht, in: Flusseinzugsgebietsmanagement und Sozioökonomie. Konfliktbewertung und Lösungsansätze, 
1. Sie schreibt eine grundlegende Änderung der räumlichen Organisation der Wasserwirtschaft in Europa vor: Die Bewirtschaftung der Ressourcen wird künftig in den Grenzen ihrer hydrologischen »Einzugsgebiete « (WRRL, Artikel 2, Absatz 13), nicht mehr innerhalb politischer Verwaltungseinheiten erfolgen. Damit sollen sich die organisatorischen Rahmenbedingungen für einen umfassenden und integrierten Gewässerschutz verbessern.

Dieser erweiterte Raumbezug stellt hinsichtlich des Managements in der »Flussgebietseinheit « (Definition siehe WRRL, Artikel 2, Absatz 15) der Oder eine Herausforderung an die internationale Zusammenarbeit dar: Oberund Unteranlieger müssen nun mehr als bisher z. B. die Datenerhebung (Vergleichbarkeit), Formen und Ziele der Gewässerunterhaltung und Flächenausweisung sowie den Umfang von Trinkwassergewinnung und -verbrauch untereinander abstimmen.

2. Oberflächengewässer müssen in einen guten ökologischen und chemischen Zustand versetzt werden. Für das Grundwasser wird ein guter chemischer und mengenmäßiger Zustand gefordert (Definition siehe WRRL, Artikel 2, Absatz 18-26). Möglich wird dies durch eine Stärkung des Verursacherprinzips in Kombination mit einem immissionsorientierten Ansatz: das Einwirken von Wasserverunreinigungen auf das Ökosystem Fluss, d. h., der durch Einleitungen verursachte Zustand findet stärkere Beachtung als bisher ${ }^{4}$.

3. Die Bewirtschaftung von Flussgebieten soll künftig unter stärkerer Beteiligung aller Gruppen von Betroffenen (stakeholder) erfolgen (WRRL, Artikel 14). Um dieser Anforderung entsprechen zu können, werden sich die Flussgebietsbehörden interaktiven Politikformen stellen müssen.

4. Wasserwirtschaft muss kostendeckend werden (WRRL, Artikel 9).

Wichtig ist die Klärung der Fragen, wer die Bewertung und Erfassung der Umweltqualität bezahlen und wie man mögliche Kosten der Ressourcennutzung ermitteln soll. Eine Monetarisierung von Ressourcen- und Umweltverbrauch zwischen wirtschaftlich und politisch ungleichen Partnern kann Ursache von Konflikt werden.

5. Die Richtlinie gibt den Ländern der Europäischen Gemeinschaft 15 Jahre Zeit, um ihre Oberflächengewässer und Grundwasservorkommen in einen »guten Zustand « zu versetzen (WRRL, Artikel 4, Absatz 1).

Mit ihrem Anliegen, ökologische, ökonomische und soziale Ansprüche an Wasserressourcen nachhaltiger gegeneinander abzuwägen, kann die Durchsetzung dieser WRRL durchaus zum Hindernis einer vorrangigen Wirtschaftsentwicklung in den Beitrittsländern werden. In Westeuropa haben rücksichtslose Eingriffe in Natur und Umwelt - nicht selten deren irreversible Schädigung - die Industrialisierung und Urbanisierung begleitet, vorangetrieben und raschen gesellschaftlichen Wohlstand ermöglicht. Der Versuch der EU, in den Beitrittsländern neue ökologische Schäden infolge wirtschaftlicher Entwicklung durch einen verstärkten Schutz

UFZ-Bericht Nr. 30/1999, S. 137-146.

$4 \mathrm{Vgl}$. auch »Nationale Gewässerschutzkonzeption. Aktuelle Schwerpunkte Beschluß der 107. LAWA-Vollversammlung am 20.09.1996«, hrsg. von der Länderarbeitsgemeinschaft Wasser, S. 12, unter: http://www.lawa.de. der Umwelt zu vermeiden, wird dort nicht nur mit Verständnis aufgenommen.

Die neue Festlegung zum Raumbezug von Gewässerbewirtschaftung grenzt den bestehenden Ermessensspielraum lokaler politischer Akteure erheblich ein. Für grenzüberschreitende Gewässer kommt einschränkend hinzu, dass deren Bewirtschaftung nun auf internationaler Ebene zu koordinieren ist. Das bedeutet für die Anrainerstaaten praktisch den Verzicht auf Teile ihrer Verwaltungs- und Planungshoheit: Entscheidungen zur Wasserwirtschaft, Landnutzung sowie Raum- und Umweltplanung bedürfen jetzt eines grenzüberschreitenden Konsensus. Sie müssen aber von politisch und wirtschaftlich ungleichgewichtigen Partnern getroffen werden, deren Akteure ihre innenpolitische Legitimierung v.a. aus der Vertretung eigenstaatlicher Interessen beziehen.

Trotz dieser schwierigen politischen und Rechtslage hat die EU eine zentrale Frage bei der Durchsetzung der WRRL nicht geregelt: Zwar legt die WRRL den Raumbezug für das Management grenzüberschreitender Gewässer fest. Innerhalb welchen institutionellen Rahmens, mit Hilfe welcher administrativen Strukturen diese hochgradig interdisziplinäre Aufgabe, die zudem so viele politische, wirtschaftliche, soziale, rechtliche und planerische Aspekte umfasst und außenpolitisch so schwierig ist, bewältigt werden soll, bleibt aber unklar5.

Die politischen Kompetenzen und rechtlichen Befugnisse der für die Planung von Flusseinzugsgebieten verantwortlichen Instanzen sind bisher nicht geregelt. Die Bandbreite möglicher administrativer Ansätze innerhalb Europas ist deshalb groß (WRRL, Artikel 3, Abs. 6). Für die Oder ist diese Frage nach wie vor nicht gelöst ${ }^{6}$.

In Europa wird die Bewirtschaftung von grenzüberschreitenden Seen und Fließgewässern, wenn es sich um ganze Einzugsgebiete handelt, überwiegend von multilateralen internationalen Kommissionen koordiniert. Einzelne Gewässerabschnitte im Bereich von Staatsgrenzen unterstehen der Aufsicht bilateraler Grenzkommissionen?

\section{Vgl. WRRL, Artikel 3.}

Großbritannien wollte in den früheren Entwürfen der WRRL die Einrichtung von Flussgebietsbehörden EU-weit durchsetzen. Erst nach Protesten von »Kontinentaleuropäern« - vor allem der Bundesregierung - gegen einen Eingriff in nationale Verwaltungsstrukturen wurde die Verpflichtung zur Einrichtung von Flussgebietsbehörden aus späteren Fassungen gestrichen. Vgl. Moss, Timothy: Zwischen »structural determinism« und inhaltlicher Anpassung. Institutionenwandel in England und Deutschland am Beispiel der wasserwirtschaftlichen Planung: http://www.irs-net.de. Zur Diskussion um diese Frage siehe auch: LAWA-Länderarbeitsgemeinschaft Wasser: Handlungskonzept zur Umsetzung der Wasserrahmenrichtlinie, Abschnitt 5: Koordination und Organisation, S. 10/11 unter: http://www.lawa.de.

6 Schriftliches Interview mit Leopold Zubek, Geschäftsführer der IKSO am 17.04.2002.

7 Deutschland ist Mitglied in mehreren internationalen Flussgebietskommissionen, deren Tätigkeit auf völkerrechtlich verbindlichen Übereinkommen beruht. Dies gilt für Rhein, Donau, Elbe, Mosel/Saar, Maas, Oder (und Bodensee). Darüber hinaus hat die Bundesrepublik Deutschland das im Rahmen der UN-Wirtschaftskommission für Europa (ECE) erarbeitete völkerrechtliche Übereinkommen zum Schutz und zur Nutzung grenzüberschreitender Wasserläufe und internationaler Seen (ECE Gewässerkonvention 1992) 1994 ratifiziert. Mit diesem Abkommen wird eine gemeinsame Grundlage zum modernen Schutz der grenzüberschreitenden Flüsse und Seen insbesondere für die Staaten Mittel- und Osteuropas geschaffen. Für das grenzüberschreitende Gewässermanagement im Odereinzugsgebiet 
Das Aufsichtsgremium für die Oder ist die am 11. April 1996 gegründete Internationale Kommission zum Schutz der Oder vor Verunreinigungen (IKSO) ${ }^{8}$. Die IKSO ist für das gesamte Einzugsgebiet der Oder zuständig. In ihren Gremien ist neben den Anrainerstaaten auch die EU vertreten. Hauptaufgabe der Kommission ist es, gemeinsame Strategien und grenzüberschreitende Programme zu erarbeiten, um so politische Entscheidungen vorzubereiten. Es wird angestrebt, die IKSO als Administration zu nutzen, die die Ausarbeitung eines internationalen Bewirtschaftungsplanes für das Einzugsgebiet der Oder entsprechend den Vorgaben der EU-WRRL koordiniert und dessen Realisierung überwacht. Dazu wurde zusätzlich eine ständige Arbeitsgruppe (6) gebildet. Bisher liegt die Zustimmung zur Wahrnehmung dieser Aufgabe nur von Seiten der Bundesrepublik und der Tschechischen Republik vor?.

sind zur Zeit die folgenden internationalen Verträge verbindlich:

(1) Vertrag zwischen der Republik Polen und der Regierung der früheren Tschechoslowakischen Republik über die Zusammenarbeit auf dem Gebiet der Wasserwirtschaft auf den Grenzgewässern vom 21.05.1958.

(2) Vertrag zwischen der Bundesrepublik Deutschland und der Republik Polen über die Zusammenarbeit auf dem Gebiet der Wasserwirtschaft auf den Grenzgewässern vom 19.05.1992.

(3) Vertrag zwischen der Bundesrepublik Deutschland und der Tschechischen Republik über die Zusammenarbeit auf dem Gebiet der Wasserwirtschaft auf den Grenzgewässern vom 12.12.1995.

(4) Vertrag zwischen der Republik Polen, der Bundesrepublik Deutschland, der Tschechischen Republik sowie der Europäischen Union über die Internationale Kommission zum Schutz der Oder gegen Verunreinigung (IKSO) vom 11.04.1996.

Im Rahmen der drei erstgenannten Verträge wurden die polnisch-tschechische, deutsch-polnische und die deutsch-tschechische Grenzgewässerkommission gebildet. Sie befassen sich unter anderem mit dem Hochwasserschutz, der Koordination der Zusammenarbeit der hydrometeorologischen Dienste, dem Austausch von Beobachtungsergebnissen und täglicher Informationen einschließlich der hydrologischen Prognosen im Hochwasserfall. Diese Grenzgewässerkommissionen sind also wichtige Gremien für den bilateralen Informationsaustausch.

8 Der am 11. April 1996 in Wrozlaw (Polen) unterzeichnete »Vertrag über die Internationale Kommission zum Schutz der Oder gegen Verunreinigung « (Oderschutz-Konvention) ist nach Ratifikation durch die vier Vertragsparteien Deutschland, Polen, Tschechien und die Europäische Gemeinschaft am 28. April 1999 in Kraft getreten. Die erste Sitzung der Delegationsleiter der Oderschutz-Kommission nach Inkrafttreten des Vertrages fand am 22./23. Juli 1999 in Wroclaw statt, dort hat auch das Sekretariat der Kommission seinen Sitz. Im Mittelpunkt der Beratungen stand die Organisation der Kommissionsarbeit. Es wurden folgende $\mathrm{Ar}-$ beitsgruppen gebildet:

Arbeitsgruppe 1: Aktionsprogramm

Arbeitsgruppe 2: Außergewöhnliche Verunreinigungen

Arbeitsgruppe 3: Rechts- und Organisationsfragen

Arbeitsgruppe 4: Hochwasser

Arbeitsgruppe 5: Ökologie/Naturschutz, später kam die

Arbeitsgruppe 6: Umsetzung der WRRL, hinzu.

Die IKSO hat inzwischen ein Sofortprogramm zum Schutz der Oder gegen Verunreinigung für den Zeitraum 1997 bis 2002 verabschiedet. Das Programm umfasst den Bau bzw. die Modernisierung von 86 kommunalen Kläranlagen und 52 Industriekläranlagen. Im Jahr 2000 legte die Kommission einen ersten Zwischenbericht über die Umsetzung des Sofortprogramms vor. Der Bericht zeigt, dass bisher zwar erste Ergebnisse erreicht werden konnten, die hochgesteckten Ziele des Programms aber verfehlt wurden und es weiterer Unterstützung bedarf, um die noch ausstehenden Aufgaben zu erfüllen. Außerdem hat die IKSO einen »Internationalen Warn- und Alarmplan Oder« vorgelegt. Im Bereich des Hochwasserschutzes wurden eine »Gemeinsame Strategie und Grundsätze für das Aktionsprogramm Hochwasserschutz im Einzugsgebiet der Oder« vereinbart, auf deren Basis ein Aktionsprogramm Hochwasserschutz vorbereitet wird.

9 Interview mit L. Zubek, Geschäftsführer der IKSO, am 17.04.2002.
Das Einzugsgebiet der Oder wird von ca. 15 Mio. Menschen bewohnt und bedeckt eine Fläche von $118.800 \mathrm{~km}^{2}$. Davon liegen 89 Prozent in Polen, 6 Prozent in der Tschechischen Republik und nur 5 Prozent in Deutschland. Bevor er auf polnischer Seite in die Ostsee fließt, bildet der Strom auf etwa $162 \mathrm{~km}$, von der Mündung der Neiße in die Oder stromabwärts bis etwa Gryfino, die deutsch-polnische Grenze.

Heute zählt das Einzugsgebiet des Flusses zu den bedeutendsten Wachstumsregionen im erweiterten EU-Wirtschaftsraum. Zugleich ist es - mit seinen naturnahen Regionen und Reservaten - ein europäisches Naturerbe von hohem Rang und ein schützenswerter ökologischer Korridor.

Aus dieser Polarisierung potenzieller Nutzungsinteressen erwächst ein beträchtliches Konfliktpotenzial, das nur im Konsens der Anrainer entschärft werden kann.

Noch dominieren im Einzugsgebiet der Oder in allen Anrainer-Staaten Land- und Forstwirtschaft. In den Volkswirtschaften der Republiken Polen und Tschechien vollzog sich aber mit dem gesellschaftlichen Umbruch seit Beginn der 90er Jahre ein tiefgehender Strukturwandel. In dessen Folge und beschleunigt durch den Beitritt dieser Staaten zur EU wird es zu einem weiteren Wachstum der Wirtschaft, vor allem aber in Industrie und Infrastruktur, kommen. Zwei damit verbundene Tendenzen werden Einfluss auf den Zustand von Flusseinzugsgebieten, darunter auch des Ökosystems der Oder, haben:

1. eine veränderte Landnutzung: weniger agrarische und stärkere industrielle Nutzung, zunehmende Urbanisierung;

2. eine zunehmende Intensivierung der Landwirtschaft in Vorranggebieten und die Nutzungsaufgabe in benachteiligten Gebieten.

Aus der Sicht des Gewässerschutzes werden sich beide Tendenzen negativ auf den ökologischen Zustand des Flusssystems auswirken.

Sehr einschneidend werden die angestrebte Intensivierung des Kohle- und Erzbergbaus und die Förderung ihrer verarbeitenden Industrien im Raum Katowice, Glogow und Ostrawa, der Ausbau des Europäischen Energieverbundnetzes und ein geplanter Transportkorridor auf und entlang der Oder das Antlitz dieses Flusssystems verändern.

Vor allem im Zusammenhang mit der Durchsetzung der europäischen WRRL sind bereits Konflikte sichtbar zwischen diesen (nationalen) Ambitionen beschleunigter wirtschaftlicher Entwicklung und dem von der WRRL vorgegebenen Ziel einer nachhaltigen (internationalen) Flussgebietsbewirtschaftung.

Es ist nicht auszuschließen, dass dabei tieferliegende Konflikte, wie z. B. die besondere Geschichte der deutsch-polnischen und der deutsch-tschechischen Beziehungen, neu entstehende ökonomische und ökologische Konflikte zusätzlich politisch aufladen werden. Auch ist es wahrscheinlich, dass Konflikte, die ihren Ursprung in wirtschaftlicher Konkurrenz haben, als ökologische Konflikte etikettiert werden, um den Beistand der umweltbewussten Öffentlichkeit gewinnen und instrumentalisieren zu können. Hierbei ist diejenige Seite, die die hohen Standards europäischen Umweltschutzes mitformuliert hat (Deutschland), zugleich in der wirtschaftlich und politisch stärkeren Position und deshalb doppelt im Vorteil. 
Aktuelles Beispiel für ein Entwicklungsvorhaben mit großem Konfliktpotenzial ist das Programm »Dla Odra 2006 «10. Mit diesem Projekt verfolgt die polnische Regierung zwei Ziele:

1. Die Verbesserung des Hochwasserschutzes auf polnischer Seite durch eine Erhöhung der Deiche und andere Maßnahmen.

2. Den Ausbau der Oder zu einer ganzjährig schiffbaren Wasserstraße von europäischem Rang: Mit einem späteren Verbindungskanal zur Donau soll ein »osteuropäischer Transportkorridor « geschaffen werden.

Aus polnischer Sicht ist die Verwirklichung dieser Ziele von essentieller Bedeutung für die wirtschaftliche Entwicklung des Landes. Beide Strategien werden aber einen unmittelbaren Einfluss auf die Situation in den Oder-nahen Regionen in Deutschland haben.

Während der Flutkatastrophe von 1997 hatte die Oder in Polen 700.000 Hektar Land unter Wasser gesetzt (in Brandenburg: 6.000 Hektar) und einige Zehntausend Häuser zerstört (in Brandenburg: 178). Dutzende Menschen starben, viele Hundert wurden verletzt. Die Sachschäden wurden in Polen mit 5 Mrd. Mark angegeben (in Brandenburg: 648 Mio. Mark). Fachleute sind sich einig in der Einschätzung, dass die zerstörerische Wirkung des Hochwassers in Brandenburg weitaus größer gewesen wäre, wenn der Hochwasserschutz im Nachbarland besser funktioniert hätte. Umgekehrt lässt das den Schluss zu, dass ein verbesserter Hochwasserschutz am polnischen Oberlauf, wie er mit dem Programm «Dla Odra 2006 « angestrebt wird, die geltenden Sicherheitsstandards in Deutschland im Krisenfall noch einmal auf den Prüfstand stellen und im Ergebnis zusätzliche finanzielle Aufwendungen erzwingen wird. Die 1997 in Polen noch existierenden natürlichen und dort durch Deichbrüche zusätzlich entstandenen Überflutungsflächen stünden bei sicheren Deichsystemen in dem Umfang nicht mehr zur Verfügung. Eine Flutwelle würde sich Ausbruchsmöglichkeiten stromabwärts suchen.

Um solchen Katastrophen vorzubeugen, hatte man nach dem Hochwasser in ostdeutschen Ministerien darüber nachgedacht, dem Fluss die Ziltendorfer Niederung und weitere Teile des Oderbruchs als Überschwemmungsfläche zurückzugeben. Wirtschaftlich und ökologisch wäre das auf lange Sicht offenbar sinnvoll. Politisch aber lässt sich ein solches Vorhaben in Deutschland nicht durchsetzen. ${ }^{11}$ Dies umso weniger, als die mit dem bevorstehenden Eintritt Polens in die EU verbundene Ausweitung der EU-Gesetzgebung auf die Beitrittsländer eine Einflussnahme auf künftige Bewirtschaftungspläne des Oder-Einzugsgebiets in Polen und Tschechien in Aussicht

10 Das 1,9 Mrd. Euro teure Projekt sieht zahlreiche Flussbegradigungen und den Bau von zwei neuen Staustufen an der Oder vor, um den Verkehr von Schubverbänden bis zu 180 Meter Länge, 11,40 Meter Breite und einem Tiefgang der Schiffsklasse III (nach ersten Plänen war die Klasse $\mathrm{V}$ vorgesehen) zu ermöglichen. Ziel dieses Vorhabens ist es, die Oder mit einem späteren Verbindungskanal bis zur Donau - zu einem wichtigen Transportweg zwischen Nord- und Süd-Europa auszubauen. Der OstseeHafen Szczecin-Świnoujście am nördlichen Endpunkt dieser Wasserstraße soll damit zum bedeutendsten Umschlagplatz für den Warenverkehr zwischen dem Ost- und Südosteuropäischen Binnenland und West- und Nordeuropa werden.

11 Vgl. Projektskizze »Konzepte, Methoden und Instrumentarien für ein integriertes, nachhaltiges Flußgebietsmanagement im Unteren Oder-Einzugsgebiet« (KOMIMO), des Landesumweltamtes Brandenburg, des ZALF Müncheberg und der WASY GmbH Berlin, S. 11. stellt. Das aber könnte eine radikale Änderung der Landnutzung in Ost-Deutschland gegenstandslos machen.

Bislang erlauben nur 5 Prozent der polnischen Flüsse eine Binnenschifffahrt nach EU-Standard (in Westeuropa sind es zwischen 20 Prozent und 80 Prozent der Flüsse). Mit dem Ausbau der Oder zu einer Wasserstraße, die geltendem europäischem Standard entspricht, erhoffte sich Polen den Anschluss an den westeuropäischen Wasserverkehrsverbund. Das Verkehrsprojekt Deutsche Einheit 17 - vor allem der Havelausbau - hatte diese Hoffnungen noch bestärkt: Schließlich war mit dieser Förderung der ostdeutschen Binnenschifffahrt auch deren Anbindung an die Verkehrswege Osteuropas geplant. Aus polnischer Sicht sollte diese Integration europäischer Infrastrukturen die wirtschaftliche Erschließung des Landes beschleunigen, die Handelswege nach Osten beleben und dort neue Arbeitsplätze schaffen.

Andererseits hat eine durchgängige Schiffbarmachung der Oder wegen der damit verbundenen Begradigung und Vertiefung des Flussbettes natürlich Auswirkungen auf die Flutgefahr am (deutsch-polnischen) Unterlauf, wie sie auch den deutschen Nachbarn während der Hochwasserkatastrophe von 1997 vor Augen geführt worden war. Es ist außerdem nicht zu übersehen, dass die mit dem Flussausbau verbundenen ökonomischen Vorteile auf polnischer Seite durchaus zum wirtschaftlichen Nachteil ihrer westlichen Nachbarn werden können - und umgekehrt: Der Ausbau der Oder zu einer europäischen Wasserstrasse wird z. B. zweifellos Warenströme umleiten, Aufträge für das polnische Transportgewerbe bringen und den Standort des polnischen Ostseehafens Szczecin-Świnoujście stärken - eines ernstzunehmenden Konkurrenten des Rostocker Überseehafens.

Gegen dieses Modell zur Bewirtschaftung der Oder-Region (Entwicklungsvariante) formiert sich, unterstützt von der EUKommission ${ }^{12}$ und verschiedenen deutschen Anliegern (z. B. den brandenburgischen Ministerien für Verkehr und Umwelt), der Widerstand v.a. von Umweltschutzorganisationen, auch aus Polen ${ }^{13}$. Sie befürworten die Umwandlung der Oder-Region in einen »ökologischen Korridor Europas« (Umweltschutzvariante).

Der vorgesehene Ausbau zu einer Wasserstraße gefährde nach Ansicht der Umweltschützer einzigartige Auenlandschaften, Pflanzen- und Tierarten und erhöhe wegen der geplanten Flussbegradigungen die Hochwassergefahr. Umweltschutzverbände begrüßen zwar, dass Polen das frühere Programm »Odra 2005« nachgebessert hat und z. B. nur noch zwei

12 Siehe u. a.: Letter by Cabinet of EC President in response to letters by WWF - 8. September 2000.

13 Mehrere Verbände haben im März 2001 in Frankfurt (Oder) ein Aktionsbündnis »Zeit für die Oder« gegründet, das gemeinsam mit gleichnamigen polnischen und tschechischen Allianzen grenzüberschreitend gegen die Ausbaupläne vorgeht (siehe »Aktionsbündnis Zeit für die Oder « - Pressemitteilung vom 14.03.2001). Der Allianz gehören neben dem BUND und dem Verein für Natur und Umwelt unter anderem der Naturschutzbund Deutschland (NABU), der WWF (Initiative für das Naturschutzprojekt »Grünes Band Oder-Neiße«), die Grüne Liga, die Deutsche Umwelthilfe, der Lebuser »Verein für Natur und Umwelt « und der Landschaftspflegeverband Mittlere Oder an. In Polen war bereits 1998 eine Koalition »Zeit für die Oder« gegründet worden, die ein »mit allen Betroffenen abgestimmtes Entwicklungskonzept für die gesamte Oderregion als europäisches Natur- und Kulturerbe« fordert - gegen die »Oder als Schifffahrtsstraße europäischen Standards «. 
statt der bisher sieben Staustufen bauen will. Auch seien Teile des Programms, wie diejenigen zur Verbesserung der Wasserqualität, »sehr gut«. Zugleich wurde aber kritisiert, dass es keine nach EU-Recht vorgeschriebene Umweltverträglichkeitsprüfung gegeben habe, die Flora-Fauna-HabitatRichtlinie $^{14}$ der EU missachtet worden sei und die Amsterdamer Verträge zur nachhaltigen Entwicklung ignoriert worden wären. Sie fordern daher, das Programm »Dla Odra 2006« unter Berücksichtigung ökologischer, wirtschaftlicher und sozialer Belange umzuarbeiten: Die Oder dürfe nicht nur als Transportweg betrachtet werden. Naturschutz, umweltverträgliche Landnutzung und die Schaffung natürlicher Überflutungsflächen zum Hochwasserschutz müssten gleichrangig Berücksichtigung finden.

In dieser Auseinandersetzung hat die Bundesrepublik als derjenige Anrainerstaat, der langjähriges EU-Mitglied ist, zweifellos einen Verhandlungsvorteil: Verlangen die Aufnahmebedingungen der EU von Polen und Tschechien doch, dass sich deren wirtschaftliche und politische Entwicklungen an den Prinzipien der Nachhaltigkeit orientieren und die Umweltgesetzgebung der EU in vollem Umfang berücksichtigt wird. Welche Formen dies annehmen kann, zeigt folgendes Beispiel: Die Bestimmungen des EU-Schutzgebietsprogramms zur Ausweisung von NATURA-2000-Flächen ${ }^{15}$ und die damit verbundenen Einschränkungen der wirtschaftlichen Nutzung dieser Gebiete gehen vom Zustand in den Industrieländern Westeuropas aus. Sie berücksichtigen also die politisch anerkannten wirtschaftlichen Zwänge, historisch gewachsene Siedlungsstrukturen sowie den Status quo der Landnutzung in den bisherigen Mitgliedstaaten: Um zu retten, was noch $\mathrm{zu}$ retten ist, sind die Ansprüche an den ökologischen $\mathrm{Zu}-$ stand schützenswerter Gebiete vergleichsweise gering. In weniger industrialisierten Ländern wie Polen oder Tschechien lassen sich nach diesen Richtlinien folglich viel mehr NATURA-2000-Flächen identifizieren - in Polen wären es ca. 40 Prozent des Staatsgebiets. ${ }^{16}$ Eine komplette Ausweisung dieser Flächen würde aber die wirtschaftliche Entwicklung nicht nur der betreffenden Reviere beeinträchtigen: Da die Landnutzung hier strengen ökologischen Auflagen unterliegt, wären kurzfristig bestenfalls ökologischer Landbau und Tou-

14 Richtlinie zur Erhaltung der natürlichen Lebensräume sowie der wildlebenden Tiere und Pflanzen, Fauna-Flora-Habitat-Richtlinie, (FFH-Richtlinie 92/43/EWG, 1992) des europäischen Schutzgebietsprogramms Natura 2000 .

Wesentliche Ziele:

- Schutz der natürlichen und naturnahen Lebensräume sowie der wildlebenden Tier- und Pflanzenarten von europäischer Bedeutung.

- Ausweisung von Schutzgebieten, die zusammen mit den Vogelschutzgebieten (s. EG-Vogelschutzrichtlinie) und den Ramsar-Gebieten ein europäisches Schutzgebietssystem (Natura 2000) bilden.

15 Siehe Anm. 10

16 Nach Auskunft europäischer Umweltschutzorganisationen ließen sich $40 \%$ der Landesfläche Polen mit Verweis auf diese Bestimmungen unter Schutz stellen. Das polnische Umweltministerium hat immerhin 15\% der gesamten Landfläche als NATURA-2000-Gebiete gekennzeichnet. Während polnische Ökologen im Oder-Einzugsgebiet 39 Gebiete identifiziert haben, die der Vogelschutz- bzw. Flora-Fauna-Habitat-Richtlinie entsprechen, bestimmte das Umweltministerium in der derselben Region lediglich 13 Gebiete, die diesen EU-Maßgaben entsprechen.

(Auskunft von Krzysztof Swierkosz von der Niederschlesischen Stiftung für Nachhaltige Entwicklung/DFE), vgl. Katarzyna Rudnik: Zum Glück ist kein Geld da. Die Oder als »europäischer ökologischer Korridor «? http://www.grueneliga.de/berlin/informieren/rabe $\rightarrow$ alf/rabe@ @rchiv/08_09 _2001/glueck.html. rismus als Erwerbszweige denkbar. Das allein sind aber nicht die Perspektiven, die man sich in Polen und Tschechien von einer EU-Mitgliedschaft erhofft. In beiden Ländern erwartet man vielmehr Investitionen in Industrie und Infrastruktur, raschen wirtschaftlichen Aufschwung, Freizügigkeit von Arbeitskräften und Waren und damit steigenden Wohlstand.

Das von der IKSO entworfene Aktionsprogramm zum Hochwasserschutz ${ }^{17}$ benennt dann auch Maßnahmen, deren Durchsetzung vor allem den polnischen und tschechischen Teil der Flussgebietseinheit betreffen würde. Dort gibt es ausgedehnte und - verglichen mit der Situation in Westeuropa - noch relativ intakte Flusssysteme und Feuchtgebiete. Diese positiven ökologischen Folgen wirtschaftlicher Rückständigkeit werden nun doppelt teuer. Auf die Länder kommt einerseits eine besondere Verantwortung und ein erhöhter Ressourcenaufwand zu: Für große, grenzüberschreitende Flusseinzugsgebiete sind besondere Planungsansätze notwendig; der Erhalt bestehender und die Schaffung neuer Überflutungsflächen, die Renaturierung der Oberläufe und die Wiederaufforstung im Quellgebiet sind teuer. Strenge Naturschutzauflagen verhindern andererseits eine ungezügelte Landnutzung (z. B. die Bebauung von Überflutungsräumen), die freilich auch Arbeitsplätze schaffen und Steuereinnahmen bringen könnte.

Um die oben skizzierten Interessen- und Nutzungskonflikte präventiv bearbeiten und mittelfristig entschärfen zu können, bedarf es eines verantwortungsbewussten Konfliktmanagements. ${ }^{18}$ Im vergangenen Jahrzehnt ist innerhalb der Organisation für Sicherheit und Zusammenarbeit in Europa, des Conflict Prevention Network der Europäischen Union und in der wissenschaftlichen Literatur ein reichhaltiges Instrumentarium zur Frühwarnung und Konfliktprävention entwickelt worden. Es zielt zwar primär auf die Verhütung gewaltförmiger ethno-politischer Konflikte, kann aber auch auf Konfliktkonstellationen angewendet werden, die einen anderen Inhalt haben und nicht gewaltsam ausgetragen werden.

Frühwarnung und Konfliktprävention stellen in ihrem Tun und Unterlassen ernsthafte Interventionen in den Konfliktprozess dar, die verantwortlich gestaltet werden müssen und deshalb eine normative Referenzebene brauchen. Für unser Thema sind dies die »PE« und die »Berliner Empfehlungen $«(B E)^{19}$ des »Internationalen Runden Tischs Grenzüberschreitendes Gewässermanagement« vom September 1998.

17 Als Folge des Oder-Hochwassers von 1997 trafen sich die Umweltminister Deutschlands, Polens und der Tschechischen Republik am 4. August 1997 und beauftragten die IKSO, ein Aktionsprogramm Hochwasserschutz zu erarbeiten. Die folgenden vier Empfehlungen sollen die Kernpunkte dieses künftigen Aktionsprogramms sein:

1. Niederschläge sollen möglichst lange flächenhaft im Einzugsgebiet zurückgehalten werden.

2. Gewässer sollen soweit wie möglich naturnah umgestaltet werden.

3. Natürliche Überschwemmungsgebiete sollen erhalten und soweit wie möglich wiedergewonnen werden, dabei soll auch der künstliche Rückhalt von Hochwasserwellen geprüft werden.

4. Hochwasservorsorge und Hochwasserschutz sollen sich auch positiv auf Naturreichtum in den Ufergebieten der Flüsse auswirken.

18 Den folgenden Ausführungen liegen konzeptionelle Überlegungen meiner IFSH-Kollegen Hans J. Giessmann und Wolfgang Zellner zugrunde.

19 »Berliner Empfehlungen«, Internationaler Round Table zu grenzüberschreitendem Gewässermanagement, Berlin September 1998, in Wasser Konflikte lösen, Zukunft gestalten, Bundesministerium für wirtschaftliche Zusammenarbeit, Materialen No. 099, Bonn 1999, S. 160-173. 
Zwischen den drei Anrainerstaaten der Oder besteht eine erhebliche wirtschaftliche, Macht- und Wissens-Asymmetrie zugunsten der Bundesrepublik Deutschland (BRD) und zu Lasten der Republik Polen und der Tschechischen Republik. Im deutsch-polnischen Verhältnis wird diese Asymmetrie noch dadurch verschärft, dass die schwächere Seite über den weit größeren Anteil am Oder-Flusssystem verfügt. Die drei Asymmetrie-Ebenen sind aufs Engste miteinander verbunden: Das unterschiedliche sozioökonomische Entwicklungsniveau der drei Länder lässt erwarten, dass es zu stark ungleichzeitigen wirtschaftlichen Entwicklungs- bzw. Anpassungsprozessen und infolgedessen zu den skizzierten konfligierenden Nutzungsansprüchen an das Odersystem kommen wird. Diese zunächst sachbezogenen Nutzungskonflikte werden fast zwangsläufig dadurch politisiert, dass Polen und die Tschechische Republik Beitrittsverhandlungen mit der EU führen und in der Endphase solcher Verhandlungen meist Paketlösungen gefunden werden, die sehr verschiedene Sachgebiete miteinander verkoppeln (z. B. Ökologie, Landwirtschaft und Freizügigkeit der Arbeitskräfte usw.). Als finanzstarkes und einflussreiches Mitglied der EU kann die BRD in diesen Verhandlungen eine entscheidende Veto-Position einnehmen. Weitere Politisierungseffekte ergeben sich aus den unterschiedlichen Perzeptionen von Problemen in der Grenzregion, wie z. B. Arbeitsmigration oder Kriminalität. Dazu kommt eine auf Grund der unterschiedlichen Potenziale fast zwangsläufige Wissensasymmetrie.

Asymmetrische Macht- und Wissensverteilung bergen grundsätzlich die Gefahr einer symbolischen Aufladung und Überhöhung von Sachkonflikten in sich. Der schwächere Partner kann den Sachkonflikt dabei zur Souveränitätsfrage erklären (vgl. BE 24), mit historischem Konfliktmaterial aufladen und die Ansprüche des als stärker empfundenen Partners als »Einmischung in die inneren Angelegenheiten « zurückweisen. Wird eine derartige symbolisch-politische Position in einem Land oder mehreren Ländern von einflussreichen innenpolitischen Kräften mit Veto-Position übernommen, können nicht nur sachgerechte Nutzungskompromisse auf längere Zeit blockiert werden. Es kann auch zu Spannungen in den Gesamtbeziehungen der betroffenen Länder kommen. Angesichts des historisch belasteten deutsch-polnischen Verhältnisses, des Charakters der Oder als Grenzfluss - eines Grenzverlaufs, der von der BRD erst vor einem Jahrzehnt endgültig anerkannt wurde - und der verbreiteten polnischen Perzeption von der Oder als »unserem Fluss « besteht die erhebliche Wahrscheinlichkeit, dass sich bei Hinzukommen der oben genannten Sachkonflikte populistische Gruppierungen des Themas bemächtigen. Ein solcher Prozess könnte auch auf deutscher Seite stattfinden, beide Entwicklungen könnten sich negativ verstärken. Dasselbe gilt - abgeschwächt - für das Verhältnis der Bundesrepublik Deutschland zur Tschechischen Republik.

Eine symbolische Aufladung sachorientierter Nutzungskonflikte bezüglich des Odersystems muss also verhindert werden. Wichtig für eine rationale und kompromissorientierte Bearbeitung bestehender sowie potentieller Konflikte ist die Verbesserung des informationellen und kommunikativen Umfelds der politischen Entscheidungsträger.

Dazu wird Folgendes vorgeschlagen: (a) Frühwarnung: Frühzeitige Identifizierung von Nutzungskonflikten, der daran beteiligten Interessengruppen, Analyse des institutionellen und politischen Umfeldes einschließlich der Potentiale und Indikatoren für eine symbolische Aufladung des Konfliktes; systematische Weitergabe der Frühwarninformation.

(b) Abbau von Kompetenz- und Informationsdefiziten in einer erweiterten Partnerstruktur (vgl. BE 27).

(c) Aufbau einer gleichwertigen Kommunikationsstruktur zwischen den Kooperationspartnern und potenziellen Konfliktparteien im erweiterten Sinne.

Empfehlenswert ist der Aufbau einer Wissensbasis über Konflikte und Konfliktstrukturen im Zusammenhang mit der Nutzung der Oder, eines Informationssystems und eines Kommunikationssystems.

\section{Wissensbasis}

(a) Es ist zu überprüfen, inwieweit das in der wissenschaftlichen Literatur konzipierte, in verschiedenen internationalen Organisationen vorhandene und teilweise erprobte präventive Instrumentarium auf den spezifischen Fall von Konflikten an grenzüberschreitenden Gewässersystemen anwendbar ist.

(b) Erforderlich ist eine realistische Einschätzung der sozioökonomischen, aber auch der institutionellen und administrativen Rahmenbedingungen (vgl. BE 5), unter denen sich sachorientierte Nutzungskonflikte entwickeln. Wichtige Informationen geben hierzu die EU-Beitrittsverhandlungen Polens und der TR sowie die Ratifizierungsprozesse künftiger Beitrittsverträge v.a. in den Feldern Umweltschutz, Landwirtschaft, Industrie, Regional- und Strukturfonds und Freizügigkeit der Arbeitskräfte sowie des Gesamtpakets. Positionen relevanter Interessengruppen (Privatwirtschaft, Gebietskörperschaften, NRO, Medien) sind in die Analyse sachorientierter Konflikte einzubeziehen.

(c) Auf dieser Grundlage sollten die deutschen, polnischen und tschechischen Medien sowie die Verlautbarungen eingreifender Akteure auf Anzeichen für eine symbolische Aufladung von Sachkonflikten hin untersucht werden.

(d) Der Frühwarnfall wäre gegeben, wenn relevante politische Akteure mit potenziellen Veto-Positionen in einem der drei Länder in systematischer Form eine symbolische Aufladung von Sachkonflikten vornehmen. Da eine Frühwarnung einen ernsthaften Eingriff in den Konflikt als solchen darstellt, muss sie nach bestimmten (noch zu erarbeitenden) Regeln erfolgen und gleichzeitig und textgleich an alle involvierten Instanzen in den drei Ländern gerichtet sein.

\section{Informationssystem}

Beim Informationssystem ist zu unterscheiden zwischen der Schaffung von wissensorientierten Voraussetzungen (a) und (b) und der aktiven Durchführung von Informationsmaßnahmen (c):

(a) Notwendig erscheint die Erarbeitung von Fallstudien über die Erfahrungen, die in anderen Fällen von grenzüberschreitendem Gewässermanagement mit der Beteiligung von Interessengruppen an der Konfliktregulierung gesammelt wurden (vgl. BE 32). Ferner sind die wasserbezogenen Aktivitäten der drei Regierungen, insbesondere der Runde Tisch für die Ostsee-Region und die für 2002 
geplante Konferenz über Globale Wasserpolitik (BE 34 und 36), zu beobachten.

(b) Die Ansprechpartner in einer erweiterten Partnerstruktur, d. h. unter Einbeziehung von Gebietskörperschaften, Privatsektor, NRO und Medien (vgl. BE 27), müssen systematisch erfasst, ihr spezifischer Informationsbedarf muss ermittelt werden.

(c) Die Informationspolitik muss nach dem Prinzip der Gleichwertigkeit aller drei Länder sowie in der Absicht erfolgen, durch präventive, proaktive Informationsarbeit unproduktive Konfliktkonstellationen zu verhindern bzw. abzuschwächen. Dafür sollten phasen- und zielgruppenspezifische Einzelmaßnahmen erarbeitet werden.

3. Kommunikationssystem

Das Informationssystem hat einen Abbau von Wissensasymmetrien durch die Verteilung gleichwertiger Informationen zum Ziel, sucht dies aber durch einen asymmetrischen Pro- zess (hin zur Zielgruppe) zu erreichen. Dagegen besteht die Aufgabe des Kommunikationssystems darin, Kommunikation auf gleicher Wissens- und Statusbasis herzustellen und den top down- durch einen bottom-up-Ansatz zu ergänzen bzw. $\mathrm{zu}$ ersetzen (vgl. BE 28).

Dafür sind folgende Gesichtspunkte relevant:

(a) Auswahl von Partnern unter Einbeziehung der o.g. erweiterten Partnerstruktur. Dabei muss der Kreis der Kommunikationspartner die am Konflikt beteiligten Akteure möglichst repräsentativ abbilden. Er muss aber schon aus praktischen Erwägungen heraus enger sein als der Kreis der durch das Informationssystem erfassten Akteure.

(b) Ermittlung von Zeitfenstern im Vorfeld als möglich erachteter Konflikte bzw. im Konfliktverlauf, die für bestimmte Maßnahmen der Konfliktregulierung bzw. der Abwendung als unproduktiv erachteter Konfliktkonstellationen geeignet erscheinen.

\section{Dokumentation}

\section{Außen- und sicherheitspolitische Programme der Bundestagsparteien 2002}

\section{Aus dem Regierungsprogramm der SPD 2002-2006}

1. Deutschlands Rolle in Europa und der Welt

Verantwortung übernehmen

Eine gerechte Weltordnung

Deutschland in Europa

Unsere Bundeswehr

\section{Verantwortung übernehmen}

Deutschlands Rolle in der Welt hat sich verändert. Unsere Stimme hat an Gewicht gewonnen. Unser Rat und unsere Hilfe sind gefragt. Die Staatengemeinschaft zählt auf Deutschland, und sie kann sich auf Deutschland verlassen. Dazu haben wir mit unserer Politik entscheidend beigetragen.

Weil wir Mitverantwortung tragen für die gemeinsame Sicherheit in unserer einen Welt, haben wir die Außenpolitik, die Politik der wirtschaftlichen Zusammenarbeit und die Sicherheitspolitik schrittweise weiter entwickelt. Diesen Prozess wollen wir fortsetzen und durch die Geltung gemeinsamer Rechtsgrundsätze weltweit stärken.

Unser Ziel ist die Sicherung des Friedens weltweit. Interessengegensätze müssen friedlich ausgeglichen werden.

Mit uns wird von Deutschland niemals Aggression ausgehen. Aber die Wirklichkeit der Welt ist nicht nur friedlich. Unsere Aufgabe besteht heute in der Prävention von Konflikten und der Eindämmung von Gewalt. Wir engagieren uns für eine aktive Friedenspolitik. Dabei ergibt sich unsere Rolle in dieser Politik aus der geografischen und politischen Lage Deutschlands im Herzen Europas, unserer Mitgliedschaft in der Europäischen Union, den Vereinten Nationen und als Partner im Atlantischen Bündnis. Unsere Friedenspolitik wird bestimmt durch unsere Werte und Überzeugungen und unsere nationalen Interessen.

$\mathrm{Ob}$ auf dem Balkan, bei der Neuordnung und dem Wiederaufbau in Afghanistan, bei der Lösung regionaler Konflikte oder im Kampf gegen den internationalen Terrorismus - seit 1998 war Deutschland auch sicherheitspolitisch verstärkt gefordert und ist seiner internationalen Verantwortung gerecht geworden. Und zwar so, wie es unserem Selbstverständnis entspricht und wie unsere Partner und die Staatengemeinschaft es von uns erwarten können.

Mit der staatlichen Einheit haben wir nicht nur unsere nationale Souveränität in vollem Umfang zurückgewonnen, sondern zugleich auch das Recht und die Pflicht zur internationalen Solidarität. Wir sind ein normales europäisches Land geworden.

Wir stehen zum Einsatz unserer Soldaten international, wo er durch Beschlüsse der Vereinten Nationen und des Deutschen Bundestages legitimiert und den Soldaten gegenüber verantwortbar ist. Man darf sich seiner Verantwortung nicht entziehen, wenn Völkermord oder die Eskalation gewaltsamer Konflikte drohen oder geschehen. Das ist auch eine Lehre aus unserer eigenen Geschichte.

\section{Die Vereinten Nationen stärken}

Frieden und die weltweite Achtung der Menschenrechte sind unser Ziel. Der Weg dorthin führt über verstärkte Zusammenarbeit, über aktive Hilfe bei der wirtschaftlichen Entwicklung und ein gemeinsames System der Sicherheit und des Rechts.

Wir haben den Menschen nicht nur Märkte anzubieten, sondern auch Chancen und Solidarität.

Die Vereinten Nationen sind unverzichtbar für die Zusammenarbeit auf dieser Welt. Die Mehrzahl der 189 Mitgliedsstaaten 